\title{
ORIGINAL
}

\section{FACTORES INDIVIDUALES Y DEL ENTORNO ASOCIADOS A LA VARIABILIDAD GEOGRÁFICA DE LOS TRASTORNOS PSICOLÓGICOS ENTRE ÁREAS PEQUEÑAS: UN ANÁLISIS MULTINIVEL (*)}

\author{
Rosa Gispert Magarolas (1), Xavier Puig Oriol (2), Elisabet Palomera Fanegas (1), Jaume \\ Autonell Caldenteny (3), Josep Ma Giné Giné (1), Gloria Ribas Serra (1), José Juan Coll Fiol (1) \\ (1) Servei d'Informació i Estudis. Departament de Salut. Generalitat de Catalunya. \\ (2) Departament d'Estadística. Universitat Politècnica de Catalunya. \\ (3) Sant Joan de Deu. Serveis de salut mental. Barcelona. \\ (*) Financiado: Agència d'Avaluació de Tecnologia i Recerca Mèdiques, convocatoria 2000.
}

\section{RESUMEN}

Fundamento: Para planificar las necesidades de servicios sanitarios es fundamental conocer la distribución de la morbilidad por trastornos psicológicos en el territorio así como los factores que la determinan. El objetivo es identificar los factores que pueden explicar la variabilidad geográfica de estos trastornos en Cataluña.

Métodos: Los datos proceden de la Encuesta de Salud de 1994 y de la estadística de población de 1996 para Cataluña. Se estima la prevalencia de trastornos psicológicos por edad y sexo y por sector sanitario. En un modelo de regresión logística a dos niveles se estudia la asociación entre las variables individuales (primer nivel: Encuesta de Salud $n=12.455$ ) y las de la zona de residencia (segundo nivel: el sector sanitario, $n=46$ ) con la prevalencia de trastornos psicológicos.

Resultados: Las variables individuales que influyen en las diferencias entre sectores son: la edad (45-64 años OR:0,63 y >64 años: OR:0,22), la situación laboral (no trabaja OR:1,60) y el número de trastornos crónicos (TC) (TC=1 OR: 1,75 TC=2 OR: 2,06 TC=3-5 OR:3,36 y TC $>5$ OR: 8,9) en hombres. En las mujeres, además de estas variables (edad 25-44 años OR: 0,63 45-64 años OR:0,45 y >64 años OR: 0,32 la situación laboral no trabaja OR:1,30 y el número de trastornos crónicos (TC) TC=1 OR: $1,75 \mathrm{TC}=2 \mathrm{OR}: 2,44 \mathrm{TC}=3-5$ OR:4,09 y TC>5 OR: 11,14), influye el tipo de hogar (monoparental OR: 1,42 ). Las variables a nivel de la zona de residencia son la proporción de inmigración (hombres OR:1,55 y mujeres OR:1,68) y de desempleo (hombres OR:1,07 y mujeres OR:1,06)

Conclusiones: Los factores individuales no son suficientes para explicar la variabilidad geográfica de la prevalencia de los trastornos psicológicos, ya que también influyen las características de la zona de residencia.

Palabras clave: Trastornos mentales. Comparación transcultural. Estado de salud. Factores socioeconómicos. Factores de riesgo. Análisis multinivel. Regresión logística. Análisis de área pequeña.

Correspondencia:

Rosa Gispert

Servei d'Informació i Estudis

Departament de Salut

Travessera de les Corts 131-159

08028 Barcelona

Correo ELEctrónico: rosa.gispert@gencat.net

\section{ABSTRACT}

\section{Individual and Ecological Factors Related to the Geographical Variability of Psychological Distress among Small Areas: a Multilevel Analysis in Catalonia, Spain}

Background: To know the geographic distribution of the prevalence of psychological distress is important for mental health services planning. This study is aimed at identifying the individual factors and those related to the area of residence which may explain the geographic variability of psychological distress (by healthcare districts) in Catalonia.

Methods: The data was taken from the 1994 Catalonian Health Survey and from the 1996 Catalonia population statistics. The prevalence of psychological distress is estimated by age and sex and by healthcare district. In a two-level logic regression model, a study is made of the relationship between the individual variables (first level: health survey $n=12,455$ ) and those of the area of residence (second level: the healthcare district, $n=46$ ) to the geographic distribution of the prevalence of psychological distress.

Results: The significant variables at individual level are in men: age (45-64 years OR:0,63 y >64 years OR: 0,22), working status (no work OR:1,60), number of chronic diseases (CD) (CD=1 OR: 1,75 $\mathrm{CD}=2$ OR: 2,06 $\mathrm{CD}=3-5$ OR:3,36 and $\mathrm{CD}>5$ OR: 8,9). In women: age (25-44 years OR: 0,63 45-64 years OR:0,45 and >64 years OR: 0,32 ), working status (no work OR:1,30), number of chronic diseases $(C D=1$ OR: $1,75 \mathrm{CD}=2$ OR: 2,44 $\mathrm{CD}=3-5 \mathrm{OR}: 4,09$ and $\mathrm{CD}>5$ OR: 11,14 ), and also the kind of parental household in women (single-parental OR: 1,42). The variables at the level of the area of residence are inmigration (men OR:1,55 and women OR:1,68) and unemployment (men OR:1,07 and women OR:1,06).

Conclusions: The individual factors do not suffice to explain the geographical variability of the prevalence of psychological distress, but the characteristics of the area of residence are also important.

Key words: Mental disorders. Health status. Cross-cultural comparison. Socioeconomic factors. Risk factors. Multilevel analysis. L ogistic regresión. Small-area analysis. 


\section{INTRODUCCIÓN}

Los trastornos mentales han sido reconocidos por la OMS como unos de los problemas de salud crónicos más frecuentes en la mayor parte de sociedades desarrolladas ${ }^{1}$. Estudios previos en nuestra área han puesto de manifiesto diferencias territoriales en la situación de la salud mental ${ }^{2-3}$. Para la planificación de los servicios sanitarios es fundamental conocer cómo se distribuye la morbilidad por trastornos mentales a nivel del territorio, con el fin de valorar las necesidades de atención y en qué medida éstas están satisfactoriamente cubiertas.

Estudios epidemiológicos en diversos ámbitos y con distintos diseños han mostrado importantes variaciones geográficas en la prevalencia de los trastornos mentales, aunque aún hoy es difícil saber hasta que punto están influidas por aspectos metodológicos ${ }^{4}$. Los factores a los que se atribuyen estas diferencias se relacionan con características sociodemográficas del territorio, con la oferta de servicios sanitarios ${ }^{5-6}$ y con distintos indicadores de necesidad que señalan aspectos individuales, como las variables demográficas y socioeconómicas, antecedentes de factores estresantes y enfermedades orgáni$\operatorname{cas}^{7-9}$. Por tanto, la distinta distribución de estos factores a nivel del territorio podría explicar, al menos en parte, las diferencias observadas entre áreas geográficas.

El objetivo de este trabajo es analizar qué factores pueden explicar la distribución de la prevalencia de trastornos psicológicos a nivel de áreas pequeñas en Cataluña, teniendo en cuenta a la vez las variables a nivel individual y las del entorno, de forma que faciliten una mejor comprensión del fenómeno.

\section{MATERIAL Y MÉTODOS}

El estudio se refiere a la población general de Cataluña, una comunidad autónoma situada al noreste de España con algo más de seis millones de habitantes. Está dividida en 55 sectores sanitarios agrupados a su vez en 8 regiones sanitarias, que son las divisiones administrativas en las que está organizado el Servicio Catalán de la Salud (SCS), entidad gestora que ejerce las competencias autonómicas en la gestión y compra de servicios sanitarios.

Los datos proceden de diversas fuentes: la Encuesta de Salud de Cataluña (ESCA) de 1994, que contiene información a nivel individual de la población general no institucionalizada. Incluye, entre otros temas, una escala de medida de la disfunción psicológica, el General Health Questionnaire de Goldberg (GHQ-12) ${ }^{10}$, así como preguntas relativas a la salud general auto percibida, número de trastornos crónicos, discapacidad y características sociodemográficas de las personas encuestadas ${ }^{11}$. El tamaño de la población así como las características sociales, demográficas, y económicas de las áreas geográficas analizadas provienen de la estadística de población de $1996^{12}$. También se han utilizado los datos de la estadística de mortalidad $^{13}$ y de la Encuesta de establecimientos sanitarios en régimen de internado de Cataluña ${ }^{14}$, facilitados por el Departament de Sanitat i Seguretat Social.

La agrupación geográfica analizada ha sido el sector sanitario, adaptando en algunos casos sus límites geográficos a los de las comarcas, para poder disponer de la información de las variables procedentes de la estadística de población. De esta forma se han obtenido territorios que corresponden a $46 \mathrm{de}$ los 55 sectores del SCS. Se exceptúan los 11 sectores de la ciudad de Barcelona que se han considerado como una unidad, puesto que no es posible desagregarlos en las fuentes de datos disponibles. La distribución geográfica resultante se compone de 21 sectores con menos de 50.000 habitantes, 15 con más de 100.000, que se sitúan fundamentalmente a lo largo de la zona costera, y 10 con una población entre 50.000 y 100.000 habitantes. 
La variable dependiente del estudio, es decir, la prevalencia de trastornos psicológicos, se estima mediante el GHQ-12, que mide la disfunción psicológica. La población participante fue la mayor de 15 años $(\mathrm{n}=12.455)$. La puntuación en la escala por encima de un umbral determinado se interpreta como la probabilidad de padecer trastornos psicológicos ${ }^{10}$. En los estudios de validación realizados en España ${ }^{15}$ el punto de corte para determinar la probabilidad de tener estos trastornos es de $2 / 3$. Este punto permite crear la variable dicotómica «padecer trastornos psicológicos» (si/no). A partir de aquí se ha estimado por edad y sexo la proporción de individuos del total de la muestra que presentaban estos trastornos, y también se ha estimado la prevalencia de trastornos psicológicos por sectores sanitarios, de acuerdo al municipio de residencia de la persona encuestada. Los valores de la muestra se han ponderado en función del diseño y de la estratificación ${ }^{16}$.

Las variables relativas a la salud que se utilizan en los dos niveles del modelo proceden de la encuesta y se han utilizado a nivel individual (en el primer nivel del modelo) o agregadas por sector sanitario (en el segundo). Las variables socioeconómicas del primer nivel proceden de la encuesta de salud y las del segundo del resto de fuentes de información.

Todas estas variables explicativas se han seleccionado con el fin de representar el conjunto de factores que según la literatura revisada pueden tener una influencia en el desarrollo de trastornos mentales ${ }^{5-9}$, agrupados en los conceptos siguientes: comorbilidad, factores estresantes, sociodemográficos y económicos y de recursos sanitarios. En el anexo 1 se describen las variables utilizadas en el estudio.

Para el análisis conjunto de la información con distintos niveles de agregación (individual y por áreas geográficas), se ha utilizado el método de análisis de niveles múltiples. Una de las hipótesis de este tipo de análisis es que la estructura de los datos ha de ser jerárquica, es decir, cada nivel es una agrupación de los elementos del nivel inferior. En este trabajo se utiliza un modelo con dos niveles. El primero corresponde a los individuos de la población general residentes en cada sector $(n=12.455)$ y el segundo a las áreas geográficas: los sectores sanitarios $(n=46)$.

El análisis de niveles múltiples tiene en cuenta la no independencia entre elementos de un mismo grupo (en nuestro caso los individuos de un mismo sector sanitario) y descompone la varianza en varianza intra-grupal (nivel 1: de los individuos), y varianza intergrupal (nivel 2: entre sectores). En otras palabras, se divide la varianza en niveles de manera que es posible estimar la variabilidad entre las distintas áreas geográficas (sectores) y evaluar como ésta se va reduciendo al introducir variables tanto del primer nivel como del segundo ${ }^{17}$.

La estructura básica de un modelo multinivel es una extensión del modelo lineal generalizado, en el que es posible tratar el intercepto y la pendiente como variables aleatorias que varían de grupo en grupo. El modelo planteado en este estudio, teniendo en cuenta que los datos provienen de una encuesta transversal y que la variable respuesta es dicotómica (padecer trastornos mentales si/no), se formula bajo una regresión logística.

El análisis se ha realizado modelando por separado los dos sexos, dado que resultados anteriores ponen de manifiesto un posible comportamiento diferenciado. Se ha utilizado la macro glinmix implementada en $\mathrm{SAS}^{18}$.

\section{RESULTADOS}

La prevalencia de trastornos psicológicos por edad y sexo del conjunto de Cataluña en 
el año 1994 fue de 9,30\% en los hombres y de $15,56 \%$ en las mujeres, siendo del $12,68 \%$ para el total de la población. Por edad la prevalencia es más elevada en los grupos de edad más jóvenes (tabla 1).

En la figura 1 los mapas representan la distribución de la prevalencia de trastornos psicológicos por sectores sanitarios. Se observa una variabilidad importante entre ellos, aunque con una mayor concentración de prevalencias elevadas en las zonas del litoral.

Los valores descriptivos básicos de las variables sociodemográficas y de recursos que caracterizan los sectores sanitarios se presentan en la tabla 2, aunque su distribución geográfica analizada gráficamente (no mostrada) no presenta patrones fácilmente interpretables.

En el ajuste del modelo logístico multinivel destaca la inestabilidad de los resultados porque los modelos obtenidos varían en función de la estrategia y el orden en la introducción de las variables, debido en parte a la correlación existente entre ellas. La construcción del modelo para cada sexo se ha basado en el principio de parsimonia, para garantizar la estabilidad de los coeficientes ante la introducción de nuevas variables y en el criterio de mantener aquéllas que mejoren el modelo reduciendo la variabilidad entre sectores.

En el modelo ajustado para los hombres (tabla 3), en el nivel 1 (variables individuales) hay que destacar el menor riesgo (odds ratio inferiores a 1) de las edades avanzadas respecto a las jóvenes, el efecto protector de trabajar y el gradiente ascendente del riesgo al presentar un mayor número de trastornos crónicos. Aunque la variable «tipo de hogar» no es significativa, se ha mantenido en el modelo porque mejora sensiblemente su valor predictivo. Los indicadores del segundo nivel (sector sanitario) que mejor se adaptan al modelo han sido la inmigración y la tasa de desempleo.

En cuanto a la bondad del ajuste, en el modelo con variables sólo del primer nivel la varianza entre sectores era de $\tau_{00}=0,0809575$ en el límite de la significación, sin embargo al incluir las variables del segundo nivel se reduce esta variabilidad $\left(\tau_{00}=0,03626878\right.$, $\mathrm{p}=0,1406$ ) dejando de ser significativa la varianza entre los sectores.

Tabla 1

Prevalencia de trastornos psicológicos por sexo y grupo de edad. Cataluña, 1994

\begin{tabular}{|l|c|c|c|c|c|c|}
\hline & $\begin{array}{c}\text { Hombres } \\
\%\end{array}$ & IC & $\begin{array}{c}\text { Mujeres } \\
\%\end{array}$ & IC & $\begin{array}{c}\text { Total } \\
\%\end{array}$ & IC \\
\hline $15-19$ & 10,00 & $(7,72-12,27)$ & 17,87 & $(14,93-20,81)$ & 13,90 & $(12,03-15,77)$ \\
\hline $20-39$ & 9,82 & $(8,49-11,15)$ & 14,35 & $(12,87-15,83)$ & 12,21 & $(11,21-13,22)$ \\
\hline $40-59$ & 9,30 & $(7,94-10,66)$ & 15,20 & $(13,64-16,76)$ & 12,47 & $(11,42-13,53)$ \\
\hline $60-79$ & 8,21 & $(6,70-9,72)$ & 16,86 & $(15,05-18,67)$ & 13,08 & $(11,86-14,30)$ \\
\hline $80+$ & 8,73 & $(4,65-12,80)$ & 14,73 & $(10,99-18,47)$ & 12,52 & $(9,70-15,34)$ \\
\hline Total & 9,30 & $(8,62-9,98)$ & 15,56 & $(14,77-16,36)$ & 12,68 & $(12,15-13,21)$ \\
\hline
\end{tabular}

Fuente: escala GHQ-12. Encuesta de Salud de Cataluña. Ref.: 11. 


\section{Figura 1}

Prevalencia poblacional de trastornos psicológicos (GHQ-12) por sectores sanitarios. Cataluña, 1994
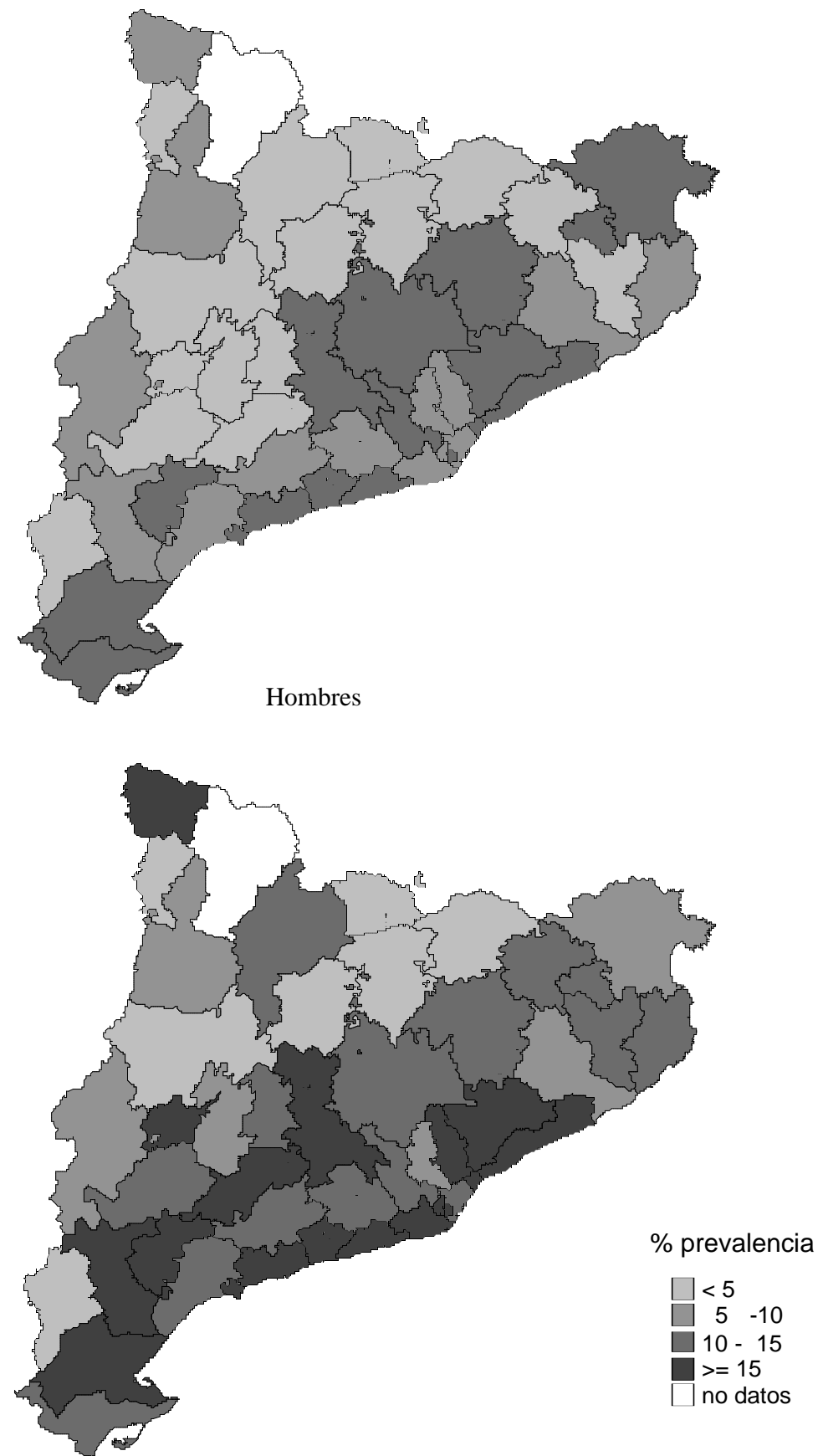

Mujeres 
Tabla 2

Descripción de las características socioeconómicas, demográficas, sanitarias y de morbilidad de los sectores sanitarios. Cataluña 1996

\begin{tabular}{|c|c|c|c|}
\hline & Total & Mínimo & Máximo \\
\hline \multicolumn{4}{|l|}{ Morbilidad $^{1}$ : } \\
\hline - trastorno psicológico (\%)* & 12,6 & 0,00 & 18,92 \\
\hline - mala salud percibida (\%) & 24,16 & 17,38 & 39,43 \\
\hline - población con alguna discapacidad (\%) & 12,00 & 5,20 & 25,33 \\
\hline - restricción de actividad (\%) & 14,4 & 0 & 17,82 \\
\hline - trastornos crónicos $(\%) *$ & 23,6 & 12,14 & 36,51 \\
\hline \multicolumn{4}{|l|}{ Factores estresantes : } \\
\hline - desocupación (\%)** & 14,82 & 7,08 & 27,12 \\
\hline - inmigración (\%) & 0,56 & 0,16 & 2,68 \\
\hline - emigración (\%) & 0,39 & 0,08 & 1,76 \\
\hline - hogares unipersonales (\%) & 4,15 & 3,22 & 5,75 \\
\hline - núcleos monoparentales (\%) & 7,37 & 4,26 & 12,05 \\
\hline - población no casada $(\%) *$ & 31,33 & 26,56 & 36,39 \\
\hline - tasa de suicidio (por 100.000 habitantes) ${ }^{2}$ & 8,31 & 0,00 & 18,26 \\
\hline \multicolumn{4}{|l|}{ Socio-económicos: } \\
\hline - densidad de población (habitantes/km2) & 195,37 & 4,29 & 20532,69 \\
\hline - mayores de 65 años (\%) & 18,51 & 10,27 & 28,09 \\
\hline - menores de 16 años $(\%)$ & 15,86 & 12,34 & 18,33 \\
\hline - renta familiar disponible (millones de ptas. x 1000 hab.) & 1565,13 & 1228,61 & 2447,01 \\
\hline - índice de dependencia & 49,78 & 36,53 & 65,11 \\
\hline - asalariados eventuales $(\%)^{* *}$ & 12,12 & 5,78 & 16,48 \\
\hline - asalariados fijos $(\%)^{* *}$ & 26,73 & 15,15 & 35,76 \\
\hline \multicolumn{4}{|l|}{ Recursos sanitarios ${ }^{2}:$} \\
\hline - camas psiquiátricas agudos (por 10.000 hab.) & 1,45 & 0,00 & 22,98 \\
\hline - médicos asociados a estas camas (por 10.000 hab.) & 0,29 & 0,00 & 3,83 \\
\hline
\end{tabular}

* Mayores de 15 años.

** 16 - 65 años.

1 Cataluña 1994.

2 Catalunya 1998.

El modelo final para las mujeres (tabla 4) es consistente con el de los hombres. A nivel individual hay que destacar la inclusión como factor explicativo del nivel de estudios y que a mayor número de trastornos crónicos supe- rior es el riesgo de presentar trastornos psicológicos. Este efecto es más acentuado que en el modelo de los hombres. Los indicadores del sector (segundo nivel) han sido la inmigración y la tasa de desempleo. La variabili- 
Tabla 3

Asociación entre la distribución territorial de la prevalencia de trastornos psicológicos (GHQ) y las características individuales y del sector de residencia, Hombres

\begin{tabular}{|c|c|c|c|c|}
\hline \multicolumn{5}{|c|}{ REGRESIÓN LOGÍSTICA MULTINIVEL } \\
\hline Efectos Fijos & OR & IC $95 \%$ & & $\mathbf{p}$ \\
\hline \multicolumn{5}{|l|}{ Nivel 1} \\
\hline Edad & & & & 0,0001 \\
\hline $15-24$ (cat.ref.) & 1,00 & & & \\
\hline $25-44$ & 1,02 & 0,74 & 1,40 & \\
\hline $45-64$ & 0,63 & 0,45 & 0,89 & \\
\hline$>64$ & 0,22 & 0,15 & 0,34 & \\
\hline \multicolumn{5}{|l|}{ Clase Social } \\
\hline I-II (cat.ref.) & 1,00 & & & 0,0764 \\
\hline III & 1,07 & 0,77 & 1,49 & \\
\hline IV-V & 1,31 & 1,00 & 1,70 & \\
\hline Laboral & & & & 0,0008 \\
\hline Trabaja (cat.ref.) & 1,00 & & & \\
\hline No trabaja & 1,60 & 1,25 & 2,04 & \\
\hline Estudiante/ama de casa & 1,15 & 0,77 & 1,71 & \\
\hline Hogar & & & & 0,3866 \\
\hline No monoparental (cat. ref.) & 1,00 & & & \\
\hline Monoparental & 1,18 & 0,81 & 1,73 & \\
\hline \multicolumn{5}{|l|}{ Trastornos crónicos } \\
\hline Ninguno (cat. ref.) & 1,00 & & & 0,0001 \\
\hline 1 & 1,75 & 1,32 & 2,33 & \\
\hline 2 & 2,06 & 1,50 & 2,84 & \\
\hline $3-5$ & 3,36 & 2,50 & 4,50 & \\
\hline+ de 5 & 8,90 & 5,98 & 13,3 & \\
\hline \multicolumn{5}{|l|}{ Nivel 2} \\
\hline Tasa de desempleo & 1,06 & 1,04 & 1,09 & 0,0001 \\
\hline$\%$ de inmigrantes & 1,68 & 1,12 & 2,50 & 0,0156 \\
\hline
\end{tabular}

\begin{tabular}{|ccc|}
\hline Efectos Aleatorios & Varianza $\left(\tau_{\mathbf{0} 0}\right)$ & p_valor \\
\hline Nivel 2 & 0,03626878 & 0,1406 \\
\hline
\end{tabular}


Tabla 4

Asociación entre la distribución territorial de la prevalencia de trastornos psicológicos (GHQ) y las características individuales y del sector de residencia, Mujeres

\begin{tabular}{|c|c|c|c|c|}
\hline \multicolumn{5}{|c|}{ REGRESIÓN LOGÍSTICA MULTINIVEL } \\
\hline \multirow{2}{*}{ Efectos Fijos } & \multirow[t]{2}{*}{ OR } & \multicolumn{2}{|c|}{ IC $95 \%$} & \multirow[t]{2}{*}{$\mathbf{p}$} \\
\hline & & & & \\
\hline Edad & & & & 0,0001 \\
\hline 15-24 (cat.ref.) & 1,00 & & & \\
\hline $25-44$ & 0,63 & 0,51 & 0,79 & \\
\hline $45-64$ & 0,45 & 0,34 & 0,58 & \\
\hline$>64$ & 0,32 & 0,24 & 0,44 & \\
\hline Nivel estudios & & & & 0,0018 \\
\hline Universitarios (cat.ref.) & 1,00 & & & \\
\hline Primarios/Secundarios & 1,30 & 0,92 & 1,82 & \\
\hline Básicos & 0,90 & 0,62 & 1,29 & \\
\hline \multicolumn{5}{|l|}{ Clase Social } \\
\hline I-II (cat.ref.) & 1,00 & & & 0,0038 \\
\hline III & 0,71 & 0,54 & 0,93 & \\
\hline IV-V & 1,04 & 0,84 & 1,29 & \\
\hline \multicolumn{5}{|l|}{ Laboral } \\
\hline Trabaja (cat.ref.) & 1,00 & & & 0,0353 \\
\hline No trabaja & 1,30 & 1,03 & 1,63 & \\
\hline Estudiante/ama de casa & 1,02 & 0,85 & 1,23 & \\
\hline \multicolumn{5}{|l|}{ Hogar } \\
\hline No monoparental (cat. ref.) & 1,00 & & & 0,0049 \\
\hline Monoparental & 1,42 & 1,11 & 1,82 & \\
\hline \multicolumn{5}{|l|}{ Trastornos crónicos } \\
\hline Ninguno (cat. ref.) & 1,00 & & & 0,0001 \\
\hline 1 & 1,75 & 1,35 & 2,27 & \\
\hline 2 & 2,44 & 1,86 & 3,19 & \\
\hline $3-5$ & 4,09 & 3,19 & 5,25 & \\
\hline+ de 5 & 11,14 & 8,34 & 14,9 & \\
\hline \multicolumn{5}{|l|}{ Nivel 2} \\
\hline Tasa de desempleo & 1,07 & 1,04 & 1,10 & 0,0001 \\
\hline$\%$ de inmigrantes & 1,55 & 1,04 & 2,32 & 0,0380 \\
\hline
\end{tabular}

\begin{tabular}{|ccc|}
\hline Efectos Aleatorios & Varianza $\left(\tau_{\mathbf{0 0}}\right)$ & $\mathbf{p}$ \\
\hline Nivel 2 & 0,10166655 & 0,0105 \\
\hline
\end{tabular}

dad entre sectores ajustando el modelo con sólo las variables individuales es estadística- mente significativa y es más del doble que la obtenida para los hombres $\left(\tau_{00}=0,19829320\right.$, 
$\mathrm{p}=0,0026$ versus $\tau_{00}=0,0809575$ en los hombres), sin embargo con la inclusión de las variables de segundo nivel la varianza del modelo final se reduce notablemente $\left(\tau_{00}=0,10166655, p=0,0105\right)$, aunque no deja de ser estadísticamente significativa.

\section{DISCUSIÓN}

Los resultados de este estudio ponen de manifiesto que en Cataluña se observa una importante variabilidad territorial en la distribución de la prevalencia de los trastornos psicológicos. La mayor frecuencia se sitúa en las zonas del litoral mediterráneo donde se concentra mayor densidad de población. Esta distribución sería consistente con la mayor frecuencia de problemas de salud mental en las zonas urbanas ${ }^{19}$. Aunque una parte de la variabilidad pueda estar justificada por la consistencia de los datos (debida a las diferencias en los efectivos de población), la variabilidad territorial ya había sido puesta de manifiesto en otros estudios realizados en la misma zona geográfica ${ }^{2-3}$.

La magnitud de la prevalencia de los trastornos psicológicos observada para el conjunto de Cataluña ${ }^{20}$ es coherente con la de otros trabajos y se encuentra en una posición intermedia con respecto a la de otros países ${ }^{4}$. Además, las diferencias entre sectores sanitarios coinciden con los resultados evidenciados a nivel de zonas más amplias, como son las regiones sanitarias, con una prevalencia alta en las regiones sanitarias de Tarragona y Tortosa (en la costa mediterránea al sur de Cataluña), baja en las de Lérida y Gerona (situadas en la parte más occidental y nororiental respectivamente) y en torno a la media en el resto (donde se sitúa la zona de Barcelona, la de mayor importancia numérica respecto al conjunto de Cataluña) ${ }^{2}$.

Del análisis de niveles múltiples destacan dos aspectos muy importantes del fenómeno estudiado. En primer lugar que los factores que pueden explicar la variabilidad territori- al pertenecen a los dos niveles de agregación (características individuales y del territorio de residencia). En segundo, que estos factores son de naturaleza diversa, tanto relacionados con la salud como con las características socioeconómicas o del entorno social.

En los modelos obtenidos para ambos sexos, las variables más relevantes son las individuales (edad, no trabajar y número de trastornos crónicos, etc.), destacando por encima de todo la morbilidad crónica. Sin embargo, la distinta distribución territorial de estos factores no explica completamente la variabilidad de la prevalencia de trastornos psicológicos entre los sectores. En cambio, al introducir las variables del segundo nivel, es decir las relativas al territorio, la variabilidad entre sectores se reduce y ambos modelos mejoran. En el segundo nivel (sector sanitario) los factores explicativos pertenecen fundamentalmente al ámbito socioeconómico (tasa de desempleo y proporción de población inmigrante). Eso quiere decir que además de un efecto individual se observa un efecto vinculado al lugar de residencia, en el sentido de que unas zonas tienen una mayor prevalencia de trastornos mentales que otras.

A pesar de las diferencias en el diseño los resultados de la literatura consultada muestran una amplia coincidencia con los de este trabajo, tanto en los factores individuales como en los de territorio. De manera general, la comorbilidad y la situación socioeconómica son los factores más importantes a nivel individual ${ }^{5-9,21}$ mientras que los factores económicos y demográficos lo son a nivel de grupo ${ }^{6,7,19}$. La asociación de un número importante de trastornos crónicos con depresión, ansiedad y otros trastornos mentales, se justifica en gran medida por el hecho de que estas personas han de adaptar sus aspiraciones, estilos de vida, situación laboral, etc. en función de su enfermedad ${ }^{22}$, lo que a su vez también tiene implicaciones socioeconómicas $^{21}$. El hecho de que los datos analizados provengan de un estudio 
transversal no permite diferenciar si los trastornos crónicos son anteriores o consecuencia del problema psicológico, pero confirman la asociación observada por otros autores $^{9,23-25}$.

Los indicadores sociodemográficos utilizados en este trabajo se han seleccionado de acuerdo a los factores que según la literatura se mostraban asociados a los trastornos mentales y teniendo en cuenta las recomendaciones de un proyecto europeo sobre este tema $^{26}$. Sin embargo la información finalmente disponible, ha determinado en gran medida el tipo de variables utilizadas, que en algunos casos puede que no reflejen el fenómeno de forma óptima.

Aparte de las limitaciones de las variables, debe hacerse mención a las limitaciones del método. Los resultados de los modelos son bastante inestables de manera que ha tenido que utilizarse un procedimiento conservador en su construcción, por ello hay que ser cuidadoso en la interpretación de los resultados. Es razonable pensar que la influencia de las variables grupales del modelo refleje su asociación con otras variables posibles y no recogidas o, incluso, la interacción de diversas variables entre sí. Por eso, tiene que hacerse una interpretación genérica de los resultados en el sentido de que la prevalencia de trastornos mentales presenta una asociación con factores socioeconómicos y demográficos a nivel de sector sanitario, más que dar importancia a las variables del modelo desempleo o emigración. Por otra parte, cabe señalar que en la actualidad se dispone de nuevos datos para estimar la prevalencia de trastornos mentales en Cataluña que facilitan estimaciones similares $^{27}$. Sin embargo, el tamaño de la muestra de la nueva encuesta no permite una desagregación por áreas pequeñas como la que aquí se ha realizado. Puesto que el objetivo del estudio es más explicativo que descriptivo, entendemos que la utilización de datos anteriores, aunque pueda ser un inconveniente, no invalida los resultados. Por otra parte, a pesar de que la muestra utilizada es muy grande, en algún territorio con un número muy pequeño de habitantes no se han obtenido casos y, a pesar de las ponderaciones realizadas, puede suponer una limitación $^{28}$.

En definitiva, los resultados de este trabajo ponen de manifiesto que la variabilidad geográfica en la presentación de los trastornos psicológicos no se explica solo con las variables de las personas, también hay que considerar variables contextuales a nivel de la zona geográfica de su residencia. Es decir, dos personas con iguales características individuales (edad, estado civil, número de trastornos crónicos, etc.) tienen una probabilidad diferente de presentar trastornos mentales si la zona donde residen es diferente (en la tasa de desempleo, de inmigración, etc.). Este hecho tiene importantes implicaciones en la planificación de servicios, dado que aconsejaría estimar la necesidad de recursos teniendo en consideración no sólo las características personales sino también las de las de la zona geográfica de referencia. 


\section{Anexo 1}

Definición de las variables utilizadas en el estudio

\section{Nivel 1: individuos}

Características individuales:(Fuente: ESCA,1994)

- Edad: años cumplidos agrupados según análisis.

- Clase Social: medida a partir de una clasificación basada en la ocupación: clase I-II (la más alta); III; IV-V.

- Situación laboral: del entrevistado, agrupada en: trabaja, no trabaja (paro, pensionista, etc.), estudiante + ama de casa.

- Tipo de hogar: agrupado según sus miembros en: monoparental y no monoparental.

- Trastornos crónicos: número de trastornos de la lista prefijada de la encuesta en categorías: ninguno, 1; 2; 3-5; >5.

Nivel 2: Sector Sanitario

Morbilidad: (Fuente: ESCA,1994)

- Trastorno psicológico: proporción de población con trastornos psicológicos (GHQ-12>2) por sexo*.

- Estado de salud: proporción de población con mala salud percibida (categorías: regular + mala).

- Discapacidad: proporción de población con alguna discapacidad de la lista.

- Restricción de actividad: proporción de población con restricción de actividad en el último año.

- Enfermedades crónicas: proporción de población con algún trastorno crónico*.

Factores estresantes: (Fuente: Estadística de Población,1996)

- Desocupación: población desocupada respecto a la población activa por $100 * *$.

- Inmigración: porcentaje de inmigrantes (del resto del país y de otros países) sobre la población de cada sector.

- Emigración: porcentaje de emigraciones sobre la población de cada sector.

- Hogares unipersonales: porcentaje de población >16 años que vive sola.

- Núcleos monoparentales: porcentaje de población >16 años que son padres solos.

- Población no casada: porcentaje de población no casada*.

- Tasa de suicidio: mortalidad por suicidio por 100.000 habitantes. (Fuente: Mortalidad, 1996-1998).

Factores socio-económicos: (Fuente: Estadística de Población, 1996)

- Densidad de población: número de habitantes por $\mathrm{km}^{2}$.

- Población mayor: porcentaje de población mayor de 65 años.

- Población joven: porcentaje de población menor de 16 años.

- Renta familiar: renta familiar disponible (millones de pesetas por 1000 habitantes)

- Índice de dependencia: porcentaje de población $<14$ años $\mathrm{y}>65$ años.

- Trabajadores eventuales: porcentaje de asalariados eventuales**.

- Trabajadores fijos: porcentaje de asalariados fijos**.

Recursos sanitarios: (Fuente: EESRI, 1998)

- Número de camas: camas psiquiátricas de agudos por 10.000 habitantes.

- Número de médicos: médicos asociados a estas camas (ajustado a 40 horas)/ 10.000 habitantes.

ESCA: Encuesta de salud de Cataluña EESRI: Encuesta de establecimientos sanitarios en régimen de internado.

(*) Mayores de 15 años.

(**) $16-65$ años. 


\section{AGRADECIMIENTOS}

Este trabajo forma parte de un proyecto de investigación financiado por la Agència d'Avaluació de Tecnologia i Recerca Mèdiques, convocatoria 2000.

\section{BIBLIOGRAFÍA}

1. World Health Organization. The world health report 2001 - mental health: new understanding, new hope. (consultada Sep 2004). Disponible en: http: www.who.int/whr/2001/en/whr01_en.pdf

2. Rajmil L, Gispert Magarolas R, Roset Gamisans M, Muñoz Rodríguez PE, Segura Benedicto A. Prevalencia de trastornos mentales en la población general de Cataluña. Gac Sanit 1998; 12(4):153-9.

3. Haro JM, Salvador-Carulla L, Cabases J, Madoz V, Vazquez-Barquero JL. Utilisation of mental health services and costs of patients with schizophrenia in three areas of Spain. Br J Psychiatry 1998; 173:334-40.

4. Wittchen HU, Jacobi F. Size and burden of mental disorders in Europe, a critical review and appraisal of 27 studies. Eur Neuropsychopharmacol 2005; 15(4):357-76.

5. Harrison J, BarrowS, Gask L, Creed F. Social determinants of GHQ score by postal survey. J Pub Health Med 1999;21 (3):283-8.

6. Stuart H. Access to physician treatment for a mental disorder: a regional analysis. Soc Psychiatr Psychiatric Epidemiol 2000; 35(2):61-70.

7. Harrison J, Barrow S, Creed F. Mental health in the north west region of England: associations with deprivation. Soc Psychiatry Psychiatr Epidemiol 1998; 33(3):124-8.

8. Martikainen P, Stansfeld S, Hemingway H, Marmot M. Determinants of socioeconomic differences in change in physical and mental functioning. Soc Sci Med 1999; 49(4):499-507.

9. Gispert R, Rajmil L, Schiaffino A., Herdman M. Sociodemographic and health-related correlates of psychiatric distress in a general population. Soc Psychiatry Psychiatr Epidemiol 2003; 38:677-83.

10. Goldberg DP, Williams P. User's guide to the General Health Questionnaire. Windsor: NFER-Nelson 1988.
11. Departament de Sanitat i Seguretat Social. Servei Català de la Salut. Enquesta de Salut de Catalunya, 1994. Barcelona: Servei Català de la Salut; 1996.

12. Estadística de Població, 1996. Institut d'Estadística de Catalunya. Disponible en: www.idescat.es/basdades/bemc/bemc.stm (Consultada Abr 2002).

13. Servei d'informació i estudis. Anàlisi de la mortalitat a Catalunya 1998. Barcelona: Generalitat de Catalunya. Departament de Sanitat i Seguretat Social; 2000.

14. Servei d'informació i estudis. EESRI 1998. Informació estadística de l'assistència hospitalària a Catalunya. Barcelona: Generalitat de Catalunya. Departament de Sanitat i Seguretat Social; 2000.

15. Muñoz Rodriguez PE. Estudio de validación predictiva del General Health Questionnaire (GHQ) en población general urbana. Anales del Fondo de Investigación Sanitaria 1993, expediente 93/0905. Madrid: FIS; 1993:221.

16. Guillen M, Juncà S, Rué M. Aragay JM. Efecto del diseño muestral en el análisis de encuestas de diseño complejo. Aplicación a la encuesta de salud de Catalunya. Gac Sanit 2000; 14(5):399-402.

17. Rice N \& Leyland A. Multilevel models: applications to health data. J Health Serv Res Policy 1996; 1(3):154-64.

18. Witte JS, Greenland S, Kimm LL. Software for hierarchical modeling of epidemiologic data. Epidemiology 1998; 9(5):563-6.

19. Kovess-Masfety V, Alonso J, de Graaf R, Demyttenaere K. A European approach to rural-urban differences in mental health: the ESEMeD 2000 comparative study. Can J Psychiatry 2005; 50(14):926-36.

20. Herrera R, Autonell J, Spagnolo E, Gispert R. Estudio epidemiológico en salud mental de la comarca del Baix Llobregat (Barcelona), fase II. Informaciones Psiquiátricas 1990; 120:111-30.

21. Wildman J. Income related inequalities in mental health in Great Britain: analysing the causes of health inequality over time. J Health Econ 2003; 22(2):295-312.

22. Turner J, Kelly B. Emotional dimensions of chronic disease. BMJ 2000; 172:124-8.

23. Phelan M, Stradins L, Morrison S. Physical health of people with severe mental illness. BMJ 2001; 322:443-4. 
24. Pennix B, Leveille S, Ferrucci L, Van Eijk J, Guralnik JM. Exploring the effect of depresion on physical disability: longitudinal evidence from the established population for epidemiologic studies of the elderly. Am J Public Health 1999; 89(9)1346-52.

25. Demyttenaere K, Bonnewyn A, Bruffaerts R, Brugha T, De Graaf R, Alonso J. Comorbid painful physical symptoms and depression: prevalence, work loss, and help seeking. J Affect Disord 2006; 92(2-3): 185-93.
26. Beecham j, Johnson S and the EPCAT Group. The European Socio-Demographic Schedule (ESDS): rationale, principles and development. Acta Psychiatr Scand 2000;102 Sup 405:33-46.

27. Salleras L, Tresserras R. La salud i els serveis sanitaris a Catalunya. La visió dels ciutadans. Barcelona: Generalitat de Catalunya. Departament de Sanitat i Seguretat Social; 2003.

28. Fernandez E, Schiaffino A. Diseños muestrales complejos. Gac Sanit 2001; 15(1):82-83. 